\title{
Spontaneous calcium responses of SF2 rat dental epithelial cells stably ex- pressing the calcium sensor G-GECO
}

\author{
Narumi Ishida ${ }^{1}$, Kaori Murata ${ }^{1}$, Takao Morita ${ }^{2}$, Shingo Semba $^{1}$, Akihiro Nezu ${ }^{1}$, and Akihiko Tanimura ${ }^{1}$ \\ ${ }^{1}$ Division of Pharmacology, Department of Oral Biology, School of Dentistry, Health Sciences University of Hokkaido, 1757 Kanazawa, \\ Ishikari-Tobetsu, Hokkaido 061-0293, Japan; and ${ }^{2}$ Department of Biochemistry, The Nippon Dental University School of Life Dentistry \\ at Niigata, 1-8 Hamaura-cho, Chuo-ku, Niigata 951-8580, Japan
}

(Received 25 June 2021; and accepted 6 July 2021)

\begin{abstract}
Genetically-encoded calcium indicators such as G-GECO are useful for studying $\mathrm{Ca}^{2+}$ responses during long-term processes. In this study, we employed a lentiviral vector and established a rat dental epithelial cell line that stably expressed G-GECO (SF2-G-GECO). $\mathrm{Ca}^{2+}$ imaging analysis under cell culture conditions revealed that SF2-G-GECO cells exhibited spontaneous $\mathrm{Ca}^{2+}$ responses, which could be classified into the following three major patterns depending on the cell density: localized $\mathrm{Ca}^{2+}$ responses at cell protrusions at a low density, a cell-wide spread of $\mathrm{Ca}^{2+}$ responses at a medium density, and $\mathrm{Ca}^{2+}$ responses in clusters of 3-20 cells at a high density. The P2Y receptor inhibitor suramin $(10 \mu \mathrm{M})$, the ATP-degrading enzyme apyrase (5 units $/ \mathrm{mL})$, and the fibroblast growth factor (FGF) receptor inhibitor FIIN-2 $(1 \mu \mathrm{M})$ decreased the frequency of spontaneous $\mathrm{Ca}^{2+}$ responses. These results indicate that ATP and FGF are involved in the spontaneous $\mathrm{Ca}^{2+}$ responses. SF2 cells differentiate into ameloblasts via interactions with mesenchymal cells. Therefore, SF2-G-GECO cells are expected to be a useful tool for studying the functions of $\mathrm{Ca}^{2+}$ responses in regulating gene expression during tooth development.
\end{abstract}

\section{INTRODUCTION}

$\mathrm{Ca}^{2+}$ is an intracellular messenger involved in the regulation of a wide range of biological processes, including contraction, secretion, gene expression, proliferation, and differentiation (Berridge 1993; Ghosh and Greenberg 1995; Barritt 1999; Kreideweiss et al. 1999). The major mechanism of $\mathrm{Ca}^{2+}$ signaling in non-excitable cells is release of $\mathrm{Ca}^{2+}$ from intracellular stores by inositol trisphosphate $\left(\mathrm{IP}_{3}\right)$, which is produced intracellularly via stimulation of $\mathrm{G}$ protein-coupled receptors and receptor tyrosine kinases

Address correspondence to: Akihiko Tanimura

Division of Pharmacology, Department of Oral Biology, School of Dentistry, Health Sciences University of Hokkaido, 1757 Kanazawa, Ishikari-Tobetsu, Hokkaido 061-0293, Japan

Tel: 0133-23-2431, Fax: 0133-23-1399

E-mail: tanimura@hoku-iryo-u.ac.jp
(Clapham 2007; Berridge 2009). In addition, $\mathrm{Ca}^{2+}$ signaling is sustained by influx of extracellular $\mathrm{Ca}^{2+}$ by store-operated $\mathrm{Ca}^{2+}$ entry (SOCE), which is activated by a decrease in the $\mathrm{Ca}^{2+}$ concentration in intracellular stores (Putney 2007).

Stim1 and Orai1, two molecules responsible for severe combined immunodeficiency, regulate SOCE by functioning as an endoplasmic reticulum $\mathrm{Ca}^{2+}$ sensor and store-operated $\mathrm{Ca}^{2+}$ channel, respectively (Feske et al. 2005; Roos et al. 2005; Prakriya et al. 2006). Deficiency in these molecules is known to be associated with a unique clinical phenotype that is characterized by defective dental enamel calcification, a defining feature of amelogenesis imperfecta type III (Feske 2011). In addition, knockout of these molecules impairs bone calcification and enamel formation, highlighting the importance of SOCE and $\mathrm{Ca}^{2+}$ signaling in tooth development (Picard et al. 2009; Robinson et al. 2012).

The dental cups, the basis of teeth, are formed 
from dental epithelial cells derived from the oral epithelium and mesenchymal cells derived from the neural crest, and tooth development proceeds through epithelial-mesenchymal interactions between these cells (Du et al. 2018). During this process, dental epithelial cells differentiate into ameloblasts, which form tooth enamel, and mesenchymal cells differentiate into odontoblasts and pulp stem cells, which form dentin and pulp (Arakaki et al. 2012). $\mathrm{Ca}^{2+}$ signaling may be involved in the regulation of cell migration, cell proliferation, and gene expression during such epithelial-mesenchymal interactions.

To visualize $\mathrm{Ca}^{2+}$ responses associated with such a slow cellular process over time, genetically encoded calcium indicators (GECIs), such as G-GECO, are particularly useful. Unlike chemically synthesized fluorescent $\mathrm{Ca}^{2+}$ indicators, GECIs do not leak out of cells or accumulate in organelles, meaning they are suitable for long-term imaging studies under cell culture conditions (Tanimura 2015). We previously reported vitamin D3-induced spontaneous $\mathrm{Ca}^{2+}$ responses of SF2 cells by performing long-term $\mathrm{Ca}^{2+}$ imaging of SF2 cells that expressed G-GECO using adenovirus vectors (Murata et al. 2016). Despite the high efficiency of gene transfer, adenovirus vectors are relatively cytotoxic and the delivered genes are transiently expressed (Russell 2000). Lentiviruses have relatively low cytotoxicity, and are used to integrate genetic material into the host genome to allow long term, stable expression of transgenes (Shai et al. 2002; Zheng et al. 2013). This property has been used for in vivo gene transfer and the establishment of cells with stable transgene expression (Shai et al. 2002; Cockrell and Kafri 2007; Zheng et al. 2018).

In this study, we utilized a lentiviral vector to create a SF2 cell line that stably expressed G-GECO as a tool to investigate the relationship between $\mathrm{Ca}^{2+}$ responses and development and differentiation, which occur over a long period of time. These cells exhibited spontaneous $\mathrm{Ca}^{2+}$ responses under cell culture conditions in the presence of serum, and ATP and fibroblast growth factor (FGF) were involved in these responses.

\section{MATERIALS AND METHODS}

Materials. The SF2 rat dental epithelial cell line was a gift from Dr. Satoshi Fukumoto (Department of Oral Health and Development Sciences, Tohoku University Graduate School of Dentistry). CMV-GGECO1.1 (Addgene plasmid \#32445) was a gift from Dr. Robert Campbell (University of Alberta,
Canada). ATP was purchased from Boehringer Mannheim (Mannheim, Germany). Gefitinib and FIIN-2 were obtained from Aobious Inc (Gloucester, MA, USA). Suramin was from Calbiochem (La Jolla, CA, USA). All other reagents were of analytical grade.

Construction of lentiviruses for G-GECO expression. Lentiviruses were constructed and prepared using the ViraPower Lentivital Expression System (Thermo Fisher Scientific, MA, USA) according to the manufacturer's instructions. Briefly, the ccdB region of the pENTR-1A entry plasmid (Thermo Fisher Scientific) was removed by digestion with EcoRI, and the $2.3 \mathrm{kbp}$ fragment was self-ligated (pENTR- $\Delta \operatorname{ccdB})$. Next, the endogenous Nhe1 site of pENTR-1A was removed and a new Nhe1 site was added upstream of the EcoRI site in the multiple cloning site of pENTR-1A using an AMAP Multi Site-directed Mutagenesis Kit (Amalgaam, Nagoya, Japan) and the following primers: 5'-ATA ACC CTA TTA CCG ATA CGCA TGG ATC TCG G-3' and 5'GGA ACC AAT TCA GCT AGC GCT GTC GAC TGG ATC CGG-3' (the introduced NheI site is underlined), according to the manufacturer's instructions. The resulting mutated pENTR-1A was referred to as pENTR- $\triangle$ Nhe + Nhe.

The pG-GECO1.1 plasmid was digested with BamHI and EcoRI, and the resulting DNA fragment containing G-GECO was inserted into the BamHI/ EcoRI sites of pENTR- $\Delta$ Nhe + Nhe. The resulting construct was referred to as pENTR-G-GECO. The G-GECO gene of the entry clone (pENTR-G-GECO) was transferred into pLenti6.3/V5-DEST (Thermo Fisher Scientific) by the LR recombination reaction to generate the pLenti-G-GECO vector. To produce recombinant lentiviruses, 293FT cells were co-transfected with pLenti-G-GECO and ViraPower Packaging Mix using Lipofectamine 2000. After 3 days of culture, the supernatant containing the lentivirus particles was collected and used in subsequent experiments. The titer of G-GECO lentiviruses (LV-GGECO) was approximately $1 \times 10^{9}$ plaque-forming units $(\mathrm{pfu}) / \mathrm{mL}$.

Cell culture and transfection. SF2 cells were cultured in Dulbecco's Modified Eagle Medium Nutrient Mixture with Ham's F12 (DMEM/F12; Gibco, Carlsbad, CA, USA) supplemented with $10 \%$ fetal bovine serum at $37^{\circ} \mathrm{C}$ in $5 \% \mathrm{CO}_{2}$. Cells were infected with LV-G-GECO at a concentration of $10^{8} \mathrm{pfu} /$ $\mathrm{mL}$, incubated for $15-20 \mathrm{~h}$, and then cultured in $\mathrm{DMEM} / \mathrm{F} 12$. Selection was not performed because 


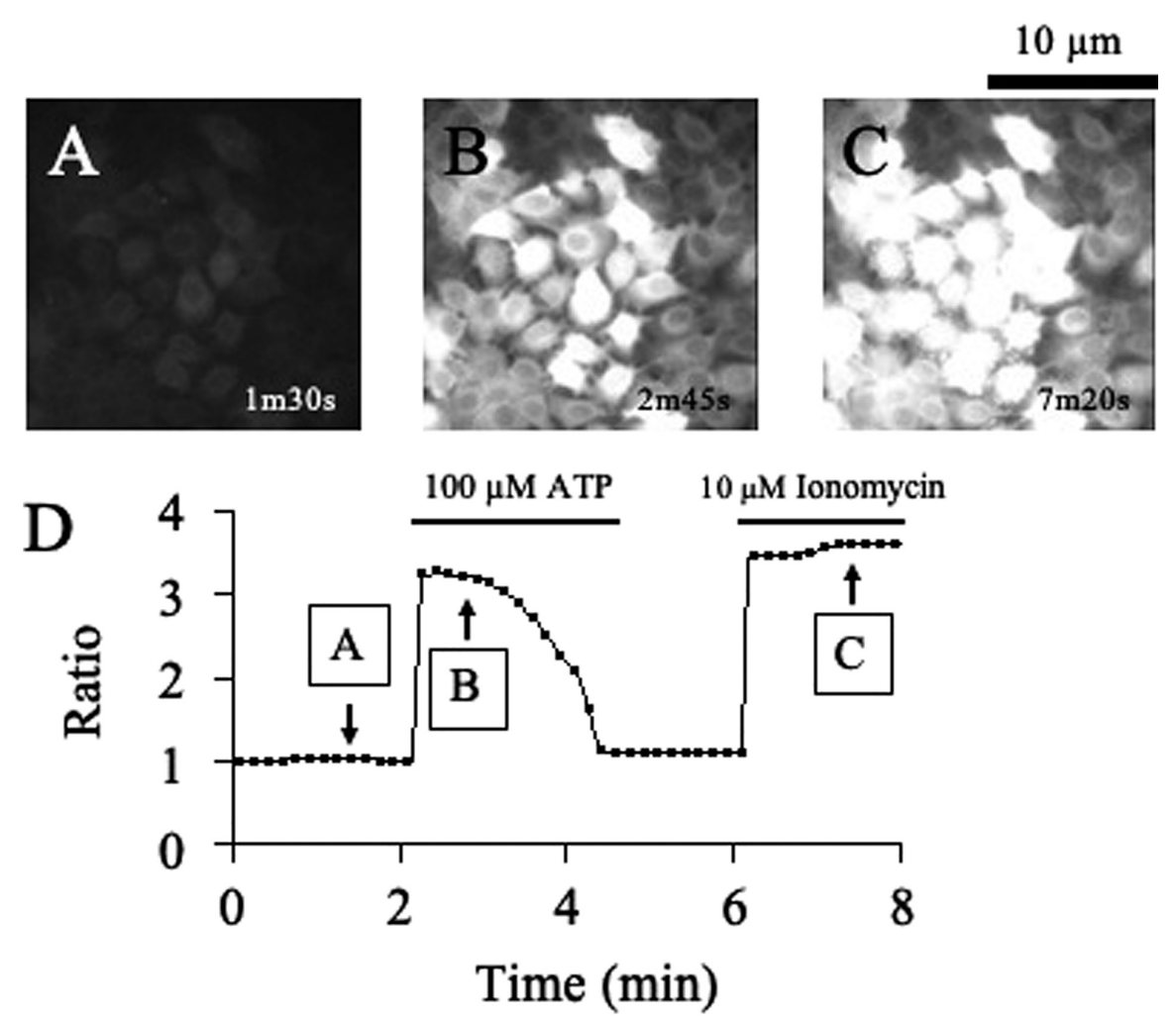

Fig. 1 ATP- and ionomycin-induced changes in fluorescence in SF2-G-GECO cells. Fluorescence images before (A) and after addition of $100 \mu \mathrm{M}$ ATP (B) and $10 \mu \mathrm{M}$ ionomycin (C), and changes in the fluorescence intensity of G-GECO (D). The presence of ATP and ionomycin is indicated by the horizontal bars at the top of the panel D. Images in A-C were captured at the time points indicated by the corresponding letters in panel D. Scale bar, $10 \mu \mathrm{m}$.

fluorescence was observed in almost all of these cells. Fluorescence was maintained in almost all cells even after ten passages.

$\mathrm{Ca}^{2+}$ imaging. G-GECO-expressing SF2 cells (SF2G-GECO cells) were cultured in DMEM/F12 lacking phenol red (Wako Pure Chemical Industries, Osaka, Japan) in fibronectin-coated chambers consisting of plastic cylinders ( $7 \mathrm{~mm}$ in diameter) attached to cover glasses. These chambers were placed on a Nikon ECLIPSE Ti2-E inverted fluorescence microscope (Nikon, Tokyo, Japan) equipped with a Nikon Fluor $\times 40$ oil immersion objective lens $(\mathrm{NA}=1.30)$ under cell culture conditions $\left(37^{\circ} \mathrm{C}, 5 \% \mathrm{CO}_{2}\right)$ during imaging using a stage chamber and a stage-and-objective lens heating system (INU-NI-F1; Tokai Hit, Shizuoka, Japan). Fluorescence images of G-GECO were captured at $30 \mathrm{sec}$ intervals using at excitation wavelength of $480 \mathrm{~nm}$ and an emission wavelength of $535 \mathrm{~nm}$ with an imaging system consisting of a DL-604M-\#VP EM-CCD camera (Andor Technology, Belfast, UK) and NIS-Elements software (Nikon).
Data analyses. Time-dependent changes in G-GECO fluorescence were analyzed using NIS-Elements imaging software (Nikon). Changes in fluorescence intensity were visualized using a ratio image, which is an image from a time point (image $\mathrm{T}$ ) divided by an image from the previous time point (image T-1) and overlaid with the fluorescent image using NIS-Elements imaging software (Nikon) and Aquacosmos imaging software (Hamamatsu Photonics, Shizuoka, Japan). Results are presented as the mean \pm standard error of the mean (SEM) of least three independent experiments. Statistical analysis was performed using Bartlett's test for homogeneity of variance followed by Fisher's least significant difference post-hoc test for comparison of individual means.

\section{RESULTS}

Fig. 1 shows changes in fluorescence of SF2-G-GECO cells associated with ATP- and ionomycin-induced increases in the intracellular $\mathrm{Ca}^{2+}$ concentration $\left(\left[\mathrm{Ca}^{2+}\right]_{\mathrm{i}}\right)$. Stimulation with ATP, which increases $\left[\mathrm{Ca}^{2+}\right]_{\mathrm{i}}$ by activating P2Y receptors, increased the 


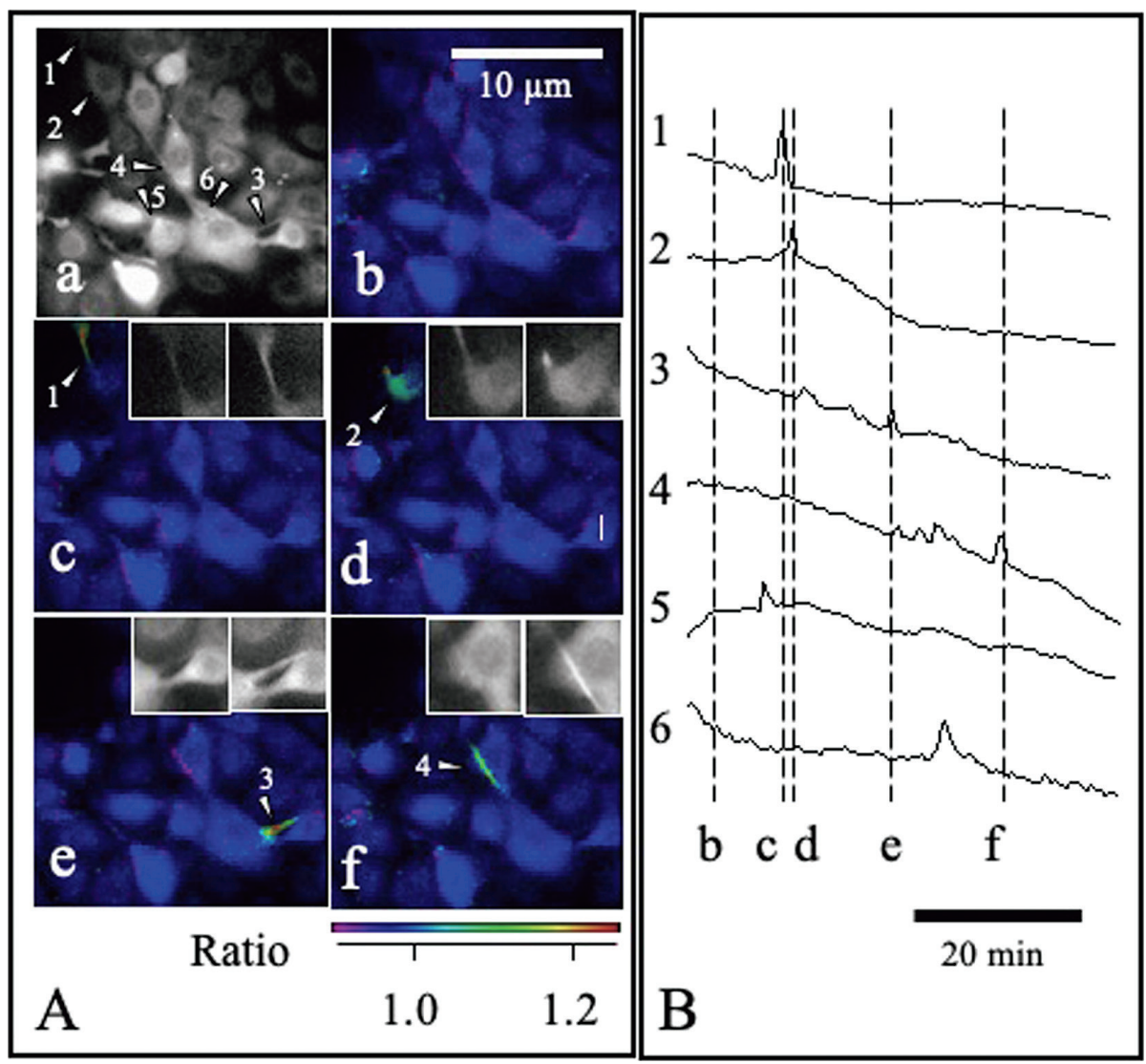

Fig. 2 Spontaneous $\mathrm{Ca}^{2+}$ responses of SF2 cells at a low density. (A) Differential interference contrast image (a) and fluorescence ratio images (b-f) at the time points indicated in panel $B$. (B) Time-dependent changes in fluorescence ratios of single SF2-G-GECO cells. Traces 1-6 in panel B are the changes in fluorescence ratios of the cells indicated by the corresponding numbers in panel Aa. Scale bar, $10 \mu \mathrm{m}$. Fluorescence of SF2-G-GECO cells was monitored at $37^{\circ} \mathrm{C}$ under cell culture conditions. $\mathrm{Ca}^{2+}$ responses were mostly observed at cell protrusions (images Ac, Ae, and Af).

fluorescence intensity in most cells, and subsequent removal of ATP returned the fluorescence intensity to the resting level. A further large increase in fluorescence intensity was observed upon application of $\mathrm{Ca}^{2+}$ ionophore ionomycin. These results showed that most SF2-G-GECO cells generated in this study expressed functional G-GECO.

$\mathrm{Ca}^{2+}$ imaging analysis under culture conditions revealed that SF2-G-GECO cells exhibited spontaneous $\mathrm{Ca}^{2+}$ responses, including $\mathrm{Ca}^{2+}$ oscillations with repeated increases in $\left[\mathrm{Ca}^{2+}\right]_{i}$ and single spikelike increases in $\left[\mathrm{Ca}^{2+}\right]_{i}$. The spatial characteristics of these $\mathrm{Ca}^{2+}$ responses can be classified into three major patterns depending on the cell density. At a low density $\left(<50\right.$ cells/frame), $\mathrm{Ca}^{2+}$ responses were observed at cell protrusions (Fig. 2). At a medium density (50-100 cells/frame), a cell-wide spread of $\mathrm{Ca}^{2+}$ responses was observed in addition to localized responses at cell protrusions (Fig. 3). At a high density $(100<$ cells/frame $), \mathrm{Ca}^{2+}$ responses were ob- served in clusters of synchronized $\left[\mathrm{Ca}^{2+}\right]_{\mathrm{i}}$ elevations in about 3-20 cells (Fig. 4c and 4f). Concordant increases in $\left[\mathrm{Ca}^{2+}\right]_{\mathrm{i}}$ in distant cells were also observed (Fig. 4d). Most of the $\mathrm{Ca}^{2+}$ responses in these clusters showed a large increases in $\left[\mathrm{Ca}^{2+}\right]_{\mathrm{i}}$. These responses in cell clusters and distant cells were considered to be caused by the diffusion of one or more bioactive substances from the cells. It was also possible that intracellular $\mathrm{Ca}^{2+}$ propagation through gap junctions may be involved in the $\mathrm{Ca}^{2+}$ response in cell clusters.

To investigate the mechanism of the spontaneous $\mathrm{Ca}^{2+}$ responses, the frequency of the increases in $\left[\mathrm{Ca}^{2+}\right]_{\mathrm{i}}$ was quantitatively analyzed under medium density conditions. It is known that ATP functions as an extracellular messenger after its release from cells in response to mechanical stimulation or an increase in $\left[\mathrm{Ca}^{2+}\right]_{\mathrm{i}}$ (Nezu et al. 2010). To investigate the involvement of ATP in the generation of these spontaneous $\mathrm{Ca}^{2+}$ responses, we examined the ef- 


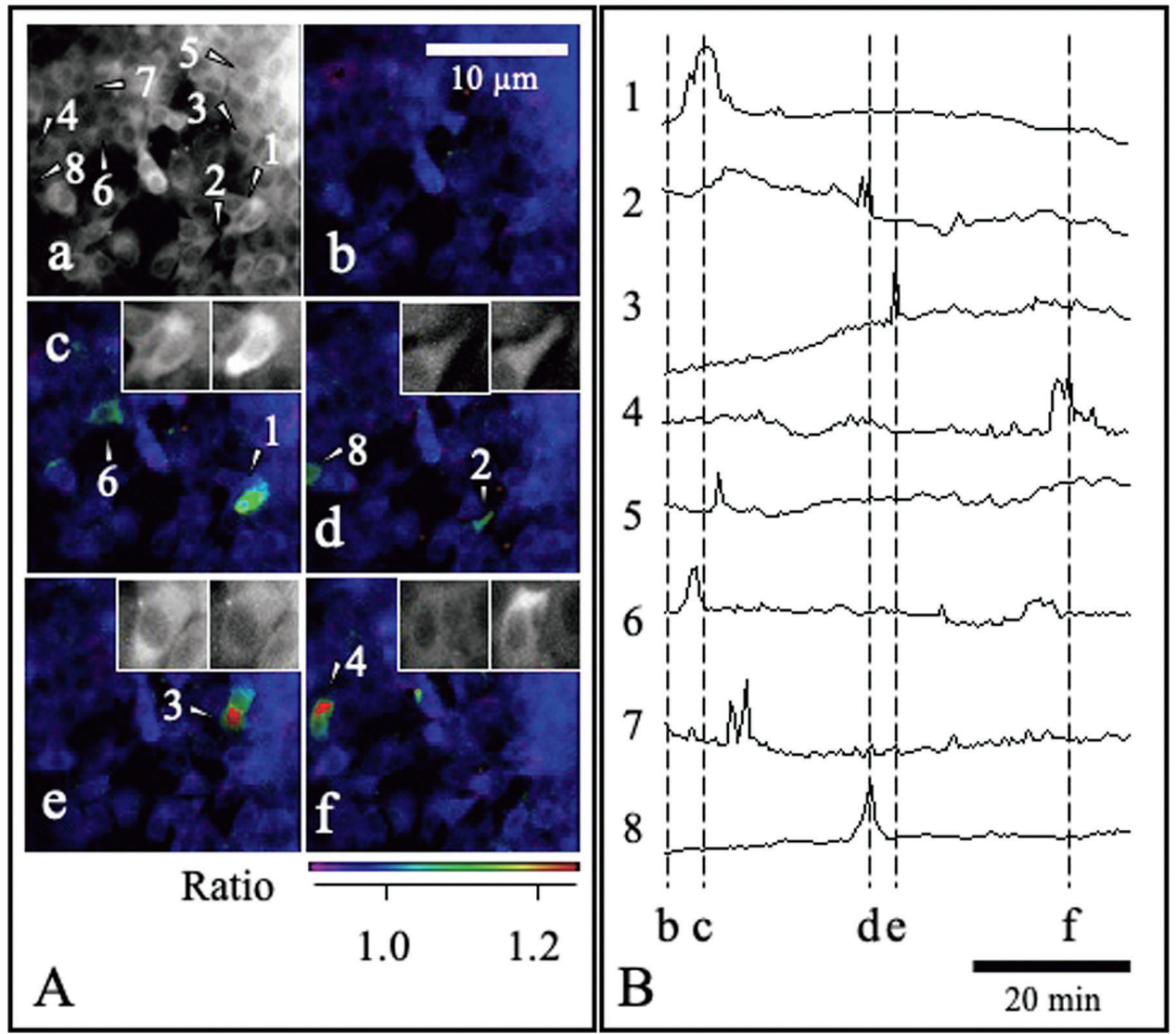

Fig. 3 Spontaneous $\mathrm{Ca}^{2+}$ responses of SF2 cells at a medium density. (A) Differential interference contrast image (a) and fluorescence ratio images $(b-f)$ at the time points indicated in panel $B$. (B) Time-dependent changes in fluorescence ratios of single SF2-G-GECO cells. Traces 1-8 in panel B are the changes in fluorescence ratios of the cells indicated by the corresponding numbers in panel Aa. Scale bar, $10 \mu \mathrm{m}$. Fluorescence of SF2-G-GECO cells was monitored at $37^{\circ} \mathrm{C}$ under cell culture conditions. $\mathrm{Ca}^{2+}$ responses were observed in whole cells (images Ac, Ae, and Af) and cell protrusions (image Ad).

fects of suramin, a P2Y receptor (P2YR) inhibitor (Wildman et al. 2003). In this experiment, we first examined $\mathrm{Ca}^{2+}$ responses under normal culture conditions (first measurement) and subsequently examined $\mathrm{Ca}^{2+}$ responses in the same area in the absence and presence of suramin (second measurement). Comparison of the first and second measurements demonstrated that the frequency of $\mathrm{Ca}^{2+}$ responses (responses/100 cells/h) in the second measurement without the inhibitor was $86.9 \%$ of that in the first measurement. The frequency of spontaneous $\mathrm{Ca}^{2+}$ responses in the second measurement was reduced to $47.8 \%$ and $52.5 \%$ of that in the first measurement upon application of 10 and $30 \mu \mathrm{M}$ suramin, respectively (Fig. 5). This result suggests that P2YR is involved in the spontaneous $\mathrm{Ca}^{2+}$ responses of SF2 cells. A further increase in the suramin concentration to $30 \mu \mathrm{M}$ did not result in more inhibition, indicating that suramin exerted its maximum inhibitory effect at $10 \mu \mathrm{M}$.

To further clarify the involvement of extracellular ATP in the spontaneous $\mathrm{Ca}^{2+}$ responses, we analyzed the effects of the ATP-degrading enzyme apyrase. Addition of apyrase ( 5 units $/ \mathrm{mL}$ ) reduced the frequency of the spontaneous $\mathrm{Ca}^{2+}$ responses to $27.9 \%$. Addition of $1 \mu \mathrm{M}$ ATP induced sustained increases in $\left[\mathrm{Ca}^{2+}\right]_{\mathrm{i}}$ or persistent $\mathrm{Ca}^{2+}$ oscillations in many SF2-G-GECO cells under cell culture conditions. These results indicate that extracellular ATP is involved in the spontaneous $\mathrm{Ca}^{2+}$ oscillations of SF2 cells. Consistent with the effect of suramin, apyrase also failed to completely inhibit $\mathrm{Ca}^{2+}$ responses, supporting the involvement of factors other than ATP.

It is known that serum contains a variety of growth factors, including epidermal growth factor (EGF) and FGF. We therefore examined the effects of the EGF receptor inhibitor Gefitinib (Wakeling et al. 2002) and the FGF receptor inhibitor FIIN-2 (Tan 


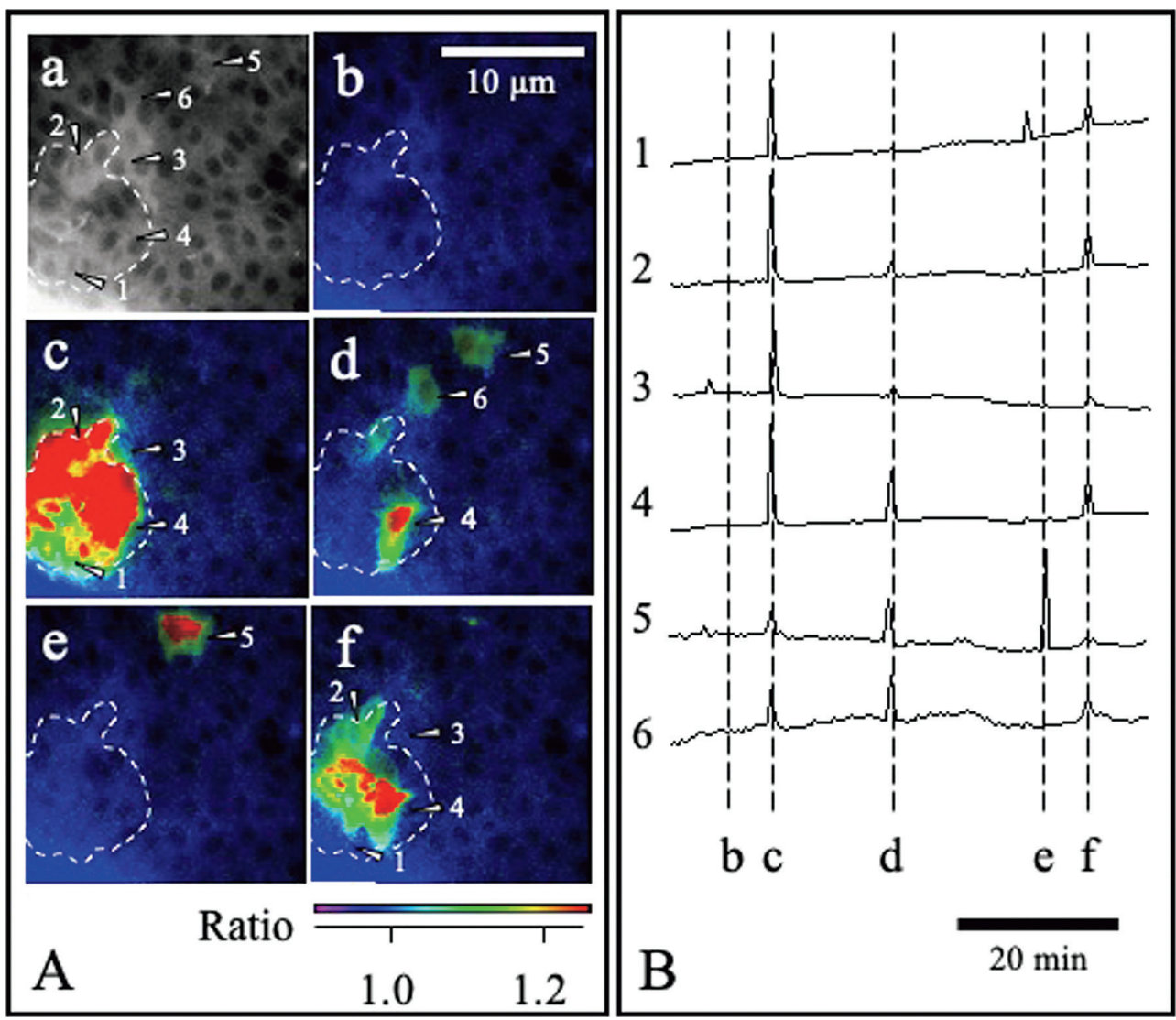

Fig. 4 Spontaneous $\mathrm{Ca}^{2+}$ responses of SF2 cells at a high density. (A) Differential interference contrast image (a) and fluorescence ratio images (b-f) at the time points indicated in panel $B$. (B) Time-dependent changes in fluorescence ratios of single SF2-G-GECO cells. Traces 1-6 in panel B are the changes in fluorescence ratios of the cells indicated by the corresponding numbers in panel Aa. Scale bar, $10 \mu \mathrm{m}$. Fluorescence of SF2-G-GECO cells was monitored at $37^{\circ} \mathrm{C}$ under cell culture conditions. $\mathrm{Ca}^{2+}$ responses were observed in cell populations (images Ac and Af) and distant cells (image Ad).

et al. 2014) on the spontaneous $\mathrm{Ca}^{2+}$ responses (Fig. 5). Gefitinib did not elicit an inhibitory effect at $1 \mu \mathrm{M}$, but reduced the frequency of spontaneous $\mathrm{Ca}^{2+}$ responses to $16.6 \%$ at $10 \mu \mathrm{M}$. In addition, FIIN-2 $(1 \mu \mathrm{M})$ reduced the frequency of spontaneous $\mathrm{Ca}^{2+}$ responses to $43.0 \%$.

\section{DISCUSSION}

In the present study, we generated SF2 cells stably expressing G-GECO (SF2-G-GECO cells) as a tool to analyze the function of $\mathrm{Ca}^{2+}$ signaling during tooth development. $\left[\mathrm{Ca}^{2+}\right]_{\mathrm{i}}$ elevation upon application of ATP and ionomycin increased fluorescence in almost all cells. These cells expressed G-GECO at a sufficient intensity and exhibited changes in fluorescence and therefore $\mathrm{Ca}^{2+}$ responses could be analyzed in the presence of serum. Serum is necessary for many cellular responses associated with development, including cell proliferation, gene expression, and mi- gration, but its weak fluorescence can interfere with the measurement cellular fluorescence. However, the fluorescence of SF2-G-GECO cells was sufficient to allow the analysis of $\mathrm{Ca}^{2+}$ responses even in the presence of serum. Thus, it was possible to detect changes in the fluorescence of SF2-G-GECO cells, and to measure $\mathrm{Ca}^{2+}$ responses in the presence of serum.

Observations of SF2-G-GECO cells under culture conditions revealed spontaneous increases in $\left[\mathrm{Ca}^{2+}\right]_{\mathrm{i}}$. The frequency of spontaneous $\mathrm{Ca}^{2+}$ responses in SF2-G-GECO cells varied greatly depending on the cell density. The frequency of spontaneous $\mathrm{Ca}^{2+}$ oscillations differed greatly depending on the area of observation and therefore we investigated the effects of inhibitors and other agents by sequentially monitoring $\mathrm{Ca}^{2+}$ responses in the same areas. The inhibitory effects of the P2YR inhibitor suramin and the ATP-degrading enzyme apyrase on the spontaneous $\mathrm{Ca}^{2+}$ responses suggest that extracellular ATP is in- 


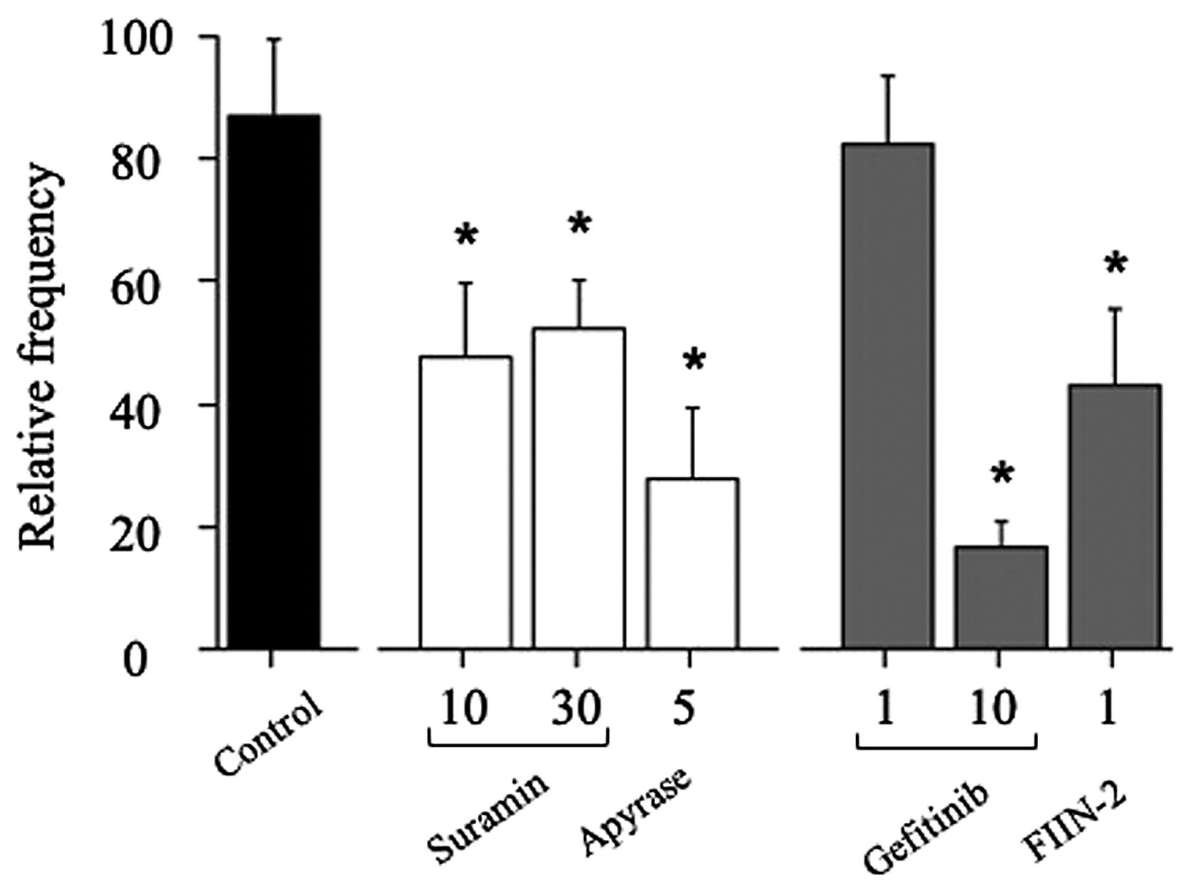

Fig. 5 Effects of inhibitors on spontaneous $\mathrm{Ca}^{2+}$ responses of SF2-G-GECO cells. $\mathrm{Ca}^{2+}$ responses were observed under cell culture conditions (first response) and subsequently in the presence or absence of inhibitors (second response). The frequencies of second $\mathrm{Ca}^{2+}$ responses relative to first $\mathrm{Ca}^{2+}$ responses are shown as percentages. The presence of suramin $(10$ and $30 \mu \mathrm{M})$, apyrase $(5$ units $/ \mathrm{mL})$, gefitinib $(1$ and $10 \mu \mathrm{M})$, and FIIN-2 $(1 \mu \mathrm{M})$ and the absence of inhibitors (control) at the second observations are indicated. Data are presented as mean \pm SEM $(n=3-25)$. Asterisks indicate significant differences from the control $\left({ }^{\star} P<0.05\right)$.

volved in the generation of these responses through P2YR. It is thought that apyrase reduced these responses by rapidly hydrolyzing the released ATP. The spontaneous $\mathrm{Ca}^{2+}$ responses of SF2-G-GECO cells were single spike-like responses with an irregular frequency. On the other hand, these cells showed sustained increases in $\left[\mathrm{Ca}^{2+}\right]_{\mathrm{i}}$ and sustained $\mathrm{Ca}^{2+}$ oscillations upon addition of extracellular ATP. Therefore, the localized and transient increase in $\left[\mathrm{Ca}^{2+}\right]_{\mathrm{i}}$ in the spontaneous responses are likely due to the hydrolysis and diffusion of ATP from SF2 cells.

The spontaneous $\mathrm{Ca}^{2+}$ responses were not completely attenuated by suramin or apyrase, suggesting that factors other than ATP are also involved. We speculate that serum growth factors such as EGF and FGF might be involved in these ATP-independent responses. The inhibitory effect of $1 \mu \mathrm{M}$ FIIN2 , an FGF receptor inhibitor, suggests that FGF is involved in the spontaneous $\mathrm{Ca}^{2+}$ responses. Gefitinib had an inhibitory effect at $10 \mu \mathrm{M}$, but not at $1 \mu \mathrm{M}$. Gefitinib specifically inhibits the tyrosine kinase activity of EGF receptor at $1 \mu \mathrm{M}$, but acts non-specifically at concentrations higher than $10 \mu \mathrm{M}$, suggesting that receptor tyrosine kinases other than the
EGF receptor are involved in the spontaneous $\mathrm{Ca}^{2+}$ responses (Wakeling et al. 2002). The present study indicates that ATP, FGF, and unidentified serum factors are involved in spontaneous $\mathrm{Ca}^{2+}$ responses in SF2-G-GECO cells. Serum contains growth factors that activate receptor tyrosine kinases (Joh et al. 1986; Wang et al. 2016), which promote $\mathrm{IP}_{3}$ production, and these are likely to be involved in the $\mathrm{Ca}^{2+}$ response.

The spontaneous $\mathrm{Ca}^{2+}$ responses of SF2 cells are likely involved in regulation of cell migration and gene expression. SF2-G-CECO cells are therefore expected to be a useful tool for direct analysis of the mechanisms that regulate these processes. Fukumoto et al. reported that co-culture of SF2 cells and dental pulp stem cells (DPSCs) leads to the differentiation of ameloblasts from SF2 cells and pulp cells from DPSCs (Eun-Jung et al. 2019). We also observed $\mathrm{Ca}^{2+}$-dependent changes in gene expression of transforming growth factor $\beta$ and Notch3 in these co-cultures (data not shown). We expect that the roles of $\mathrm{Ca}^{2+}$ signaling in the regulation of gene expression in SF2 cells and DPSCs can be explored by co-culture experiments using SF2-G-GECO cells.

Many different types of GECIs have been devel- 
oped, and most comprise the $\mathrm{Ca}^{2+}$-binding protein calmodulin $(\mathrm{CaM})$, the CaM-binding domain of myosin light chain kinase (M13), and fluorescent proteins (FPs) (Miyawaki et al. 1997; Tanimura 2015). Binding of $\mathrm{Ca}^{2+}$ to these chimeric proteins causes the M13 and CaM domains to interact and leads to a change in the fluorescence ratio or intensity (Tanimura 2015). The ratiometric response is independent of the indicator concentration, which is an important advantage of FRET-based indicators (Nagai et al. 2004; Horikawa et al. 2010). On the other hand, single FP-based indicators are advantageous for multi-color imaging in combination with other fluorescent indicators because they only require measurement of a single wavelength (Zhao et al. 2011; Chen et al. 2013).

In addition, the difference in affinity for $\mathrm{Ca}^{2+}$ is important for selection of GECIs. The high-affinity ratiometric GECI YC-Nano50, which has a $\mathrm{K}_{\mathrm{d}}$ value of $\sim 50 \mathrm{nM}$ for $\mathrm{Ca}^{2+}$ (Horikawa et al. 2010), has been used to calculate $\left[\mathrm{Ca}^{2+}\right]_{\mathrm{i}}$ at resting state (Nezu et al. 2019). By contrast, the $K_{d}$ value of G-GECO1.1 for $\mathrm{Ca}^{2+}$ is relatively high at about $620 \mathrm{nM}$ (Zhao et al. 2011), suggesting that the spontaneous $\mathrm{Ca}^{2+}$ responses of SF2 cells represent a relatively large increase in $\left[\mathrm{Ca}^{2+}\right]_{\mathrm{i}}$. In addition, G-GECO, a single FP-based indicator, facilitates simultaneous imaging with other fluorescent sensors, such as red FP-based GECIs R-GECO (Zhao et al. 2011). SF2-G-GECO cells are expected to be particularly useful when used in combination with DPSCs expressing R-GECO.

\section{Acknowledgments}

This study was supported in part by MEXT/JSPS KAKENHI grant numbers JP16H01433 and JP18H04741 "Resonance Bio", grant number 20K21686, and a Grant-in-Aid for the 2019-2020 Research Project of the Research Institute of Personalized Health Sciences, Health Sciences University of Hokkaido.

\section{REFERENCES}

Arakaki M, Ishikawa M, Nakamura T, Iwamoto T, Yamada A, et al. (2012) Role of epithelial-stem cell interactions during dental cell differentiation. J Biol Chem 287, 10590-10601.

Barritt GJ (1999) Receptor-activated $\mathrm{Ca}^{2+}$ inflow in animal cells: a variety of pathways tailored to meet different intracellular $\mathrm{Ca}^{2+}$ signalling requirements. Biochem $J 337$ (Pt 2), 153 169.

Berridge MJ (1993) Inositol trisphosphate and calcium signalling. Nature 361, 315-325.

Berridge MJ (2009) Inositol trisphosphate and calcium signalling mechanisms. Biochim Biophys Acta 1793, 933-940.
Chen T-W, Wardill TJ, Sun Y, Pulver SR, Renninger SL, et al. (2013) Ultrasensitive fluorescent proteins for imaging neuronal activity. Nature 499, 295-300.

Clapham DE (2007) Calcium Signaling. Cell 131, 1047-1058.

Cockrell AS and Kafri T (2007) Gene delivery by lentivirus vectors. Mol Biotechnol 36, 184-204.

$\mathrm{Du}$ W, Du W and Yu H (2018) The role of fibroblast growth factors in tooth development and incisor renewal. Stem Cells Int 2018, 14.

Eun-Jung K, Kyung-Sik Y, Arakaki M, Otsu K, Fukumoto S, et al. (2019) Effective differentiation of induced pluripotent stem cells into dental cells. Dev Dyn 248, 129.

Feske S (2011) Immunodeficiency due to defects in store-operated calcium entry. Ann N Y Acad Sci 1238, 74-90.

Feske S, Prakriya M, Rao A and Lewis RS (2005) A severe defect in CRAC $\mathrm{Ca}^{2+}$ channel activation and altered $\mathrm{K}^{+}$channel gating in T cells from immunodeficient patients. $J$ Exp Med 202, 651-662.

Ghosh A and Greenberg ME (1995) Calcium signaling in neurons: Molecular mechanisms and cellular consequences. Science 268, 239.

Horikawa K, Yamada Y, Matsuda T, Kobayashi K, Hashimoto M, et al. (2010) Spontaneous network activity visualized by ultrasensitive $\mathrm{Ca}^{2+}$ indicators, yellow Cameleon-Nano. Nature Methods 7, 729-732.

Joh T, Itoh M, Katsumi K, Yokoyama Y, Takeuchi T, et al. (1986) Physiological concentrations of human epidermal growth factor in biological fluids: use of a sensitive enzyme immunoassay. Clin Chim Acta 158, 81-90.

Kreideweiss S, Ahlers C, Nordheim A and Rühlmann A (1999) $\mathrm{Ca}^{2+}$-induced $\mathrm{p} 38 / \mathrm{SAPK}$ signalling inhibited by the immunosuppressant cyclosporin A in human peripheral blood mononuclear cells. Eur J Biochem 265, 1075-1084.

Miyawaki A, Llopis J, Heim R, McCaffery JM, Adams JA, et al. (1997) Fluorescent indicators for $\mathrm{Ca}^{2+}$ based on green fluorescent proteins and calmodulin. Nature 388, 882-887.

Murata K, Takahashi A, Morita T, Nezu A, Fukumoto S, et al. (2016) Effect of 1,25-dihydroxyvitamin $\mathrm{D}_{3}$ on spontaneous calcium responses in rat dental epithelial SF2 cells revealed by long-term imaging. Biomed Res (Tokyo) 37, 329.

Nagai T, Yamada S, Tominaga T, Ichikawa $\mathrm{M}$ and Miyawaki A (2004) Expanded dynamic range of fluorescent indicators for $\mathrm{Ca}^{2+}$ by circularly permuted yellow fluorescent proteins. Proc Natl Acad Sci USA 101, 10554-10559.

Nezu A, Morita T, Nagai T and Tanimura A (2019) Simultaneous monitoring of $\mathrm{Ca}^{2+}$ responses and salivary secretion in live animals reveals a threshold intracellular $\mathrm{Ca}^{2+}$ concentration for salivation. Exp Physiol 104, 61-69.

Nezu A, Tanimura A, Morita T and Tojyo Y (2010) Visualization of Ins $(1,4,5) \mathrm{P} 3$ dynamics in living cells: two distinct pathways for Ins(1,4,5)P3 generation following mechanical stimulation of HSY-EA1 cells. J Cell Sci 123, 2292-2298.

Picard C, McCarl CA, Papolos A, Khalil S, Luthy K, et al. (2009) STIM1 mutation associated with a syndrome of immunodeficiency and autoimmunity. N Engl J Med 360, 1971-1980.

Prakriya M, Feske S, Gwack Y, Srikanth S, Rao A, et al. (2006) Orail is an essential pore subunit of the CRAC channel. $\mathrm{Na}$ ture 443, 230-233.

Putney JW, Jr. (2007) Recent breakthroughs in the molecular mechanism of capacitative calcium entry (with thoughts on how we got here). Cell Calcium 42, 103-110.

Robinson LJ, Mancarella S, Songsawad D, Tourkova IL, Barnett JB, et al. (2012) Gene disruption of the calcium channel Orai1 results in inhibition of osteoclast and osteoblast differ- 
entiation and impairs skeletal development. Lab Invest 92, 1071-1083.

Roos J, DiGregorio PJ, Yeromin AV, Ohlsen K, Lioudyno M, et al. (2005) STIM1, an essential and conserved component of store-operated $\mathrm{Ca}^{2+}$ channel function. $J$ Cell Biol 169, 435445.

Russell WC (2000) Update on adenovirus and its vectors. J Gen Virol 81, 2573-2604.

Shai E, Falk H, Honigman A, Panet A and Palmon A (2002) Gene transfer mediated by different viral vectors following direct cannulation of mouse submandibular salivary glands. Eur J Oral Sci 110, 254-260.

Tan L, Wang J, Tanizaki J, Huang Z, Aref AR, et al. (2014) Development of covalent inhibitors that can overcome resistance to first-generation FGFR kinase inhibitors. Proc Natl Acad Sci USA 111, E4869-4877.

Tanimura A (2015) Development and application of fluorescent protein-based indicators for live cell imaging. J Oral Biosci $54-60$.

Wakeling AE, Guy SP, Woodburn JR, Ashton SE, Curry BJ, et al. (2002) ZD1839 (Iressa): an orally active inhibitor of epidermal growth factor signaling with potential for cancer therapy. Cancer Res 62, 5749-5754.

Wang S, Yang Q, Yu S, Pan R, Jiang D, et al. (2016) Fibroblast growth factor 1 levels are elevated in newly diagnosed type 2 diabetes compared to normal glucose tolerance controls. Endocr J 63, 359-365.

Wildman SS, Unwin RJ and King BF (2003) Extended pharmacological profiles of rat $\mathrm{P} 2 \mathrm{Y}_{2}$ and rat $\mathrm{P}_{2} \mathrm{Y}_{4}$ receptors and their sensitivity to extracellular $\mathrm{H}^{+}$and $\mathrm{Zn}^{2+}$ ions. $\mathrm{Br} J$ Pharmacol 140, 1177-1186.

Zhao Y, Araki S, Wu J, Teramoto T, Chang YF, et al. (2011) An expanded palette of genetically encoded $\mathrm{Ca}^{2+}$ indicators. Science 333, 1888-1891.

Zheng CX, Wang SM, Bai YH, Luo TT, Wang JQ, et al. (2018) Lentiviral vectors and adeno-associated virus vectors: Useful tools for gene transfer in pain research. Anat Rec (Hoboken) 301, 825-836.

Zheng L, Seon YJ, Mourão MA, Schnell S, Kim D, et al. (2013) Circadian rhythms regulate amelogenesis. Bone 55, 158-165. 